Aus dem anatomischen Institut in Bern.

\title{
Über die Beziehungen zwischen dem Blutgefässsystem und den Zellen der Nebenniere.
}

Von

Lydia Félicine.

Hierzu Tafel XI und XII.

Bei der Untersuchung der Nebennieren verschiedener Säugetiere fallt vor allem die grosse Mannigfaltigkeit der Form ihrer Zellen auf. Wenn man mit der Betrachtung an der Zona glomerulosa beginnt und allmählich gegen das Zentrum des Organes vorrückt, ist man über den schnellen Wechsel des Charakters des Protoplasmas und der Verhältnisse der Lage, Grösse und Nukleolen der Kerne geradezu überrascht. Es ist aber auch andererseits im höchsten Grade auffallend, wie sehr die feinere Struktur des Organes einer gegebenen Tierspezies z. B. des Meerschweinchens von Fall zu Fall wechselt. Man trifft hier die allerverschiedensten Bilder, wahrscheinlich je nach dem physiologischen Stadium. Desto eigentümlicher ist nun die Tatsache, dass, soweit man es heute beurteilen kann, das von der Nebenniere gelieferte Sekret in seinem chemischen Charakter und in seiner physiologischen Wirkung (Steigerung des Blutdruckes) bei verschiedenen Tieren identisch $\mathrm{zu}$ sein scheint; es können sich wenigstens die von verschiedenen Tieren stammenden Nebennierenextrakte für physiologische und pharmakologische Zwecke weit mehr gegenseitig vertreten, als es für manche andere Organsäfte der Fall zu sein scheint. Es ist daher die Schlussfolgerung unabweisbar, dass die verschiedenen Bilder und morphologischen Strukturen uns im Grunde genommen nur sehr wenig Aufschluss über den Charakter und das Wesen, geschweige denn über den Chemismus der Nebennierenzellen geben. Mögen die Zellen anscheinend auch noch so verschieden geformt und gebaut, mag das Plasma kompakt oder vakuolisiert sein, - mag sich der Kern blass oder dunkel färben, mag er einen oder mehrere Nukleolen besitzen, so haben doch alle hier in Betracht kommenden Zellen etwas spezifisches, allen gemeinsames in sich; sie verrichten vermöge 
einer unserer Wahrnehmung entgehenden Einrichtung die gleiche Arbeit, liefern überall ein wenn nicht identisches, so doch wenigstens sehr ähnliches Sekret. Die Zellen der Nebenniere scheinen somit ein klassisches Bild dafür zu liefern, wie wenig die funktionellen Verrichtungen der Zellen sich in ihrem gröberen Bau abzuspiegeln brauchen. Der ausserordentliche Formwechsel der Nebennierenzellen trăgt meiner Ansicht nach die hauptsäcbliche Schuld an den vielfachen Kontroversen über die vermutliche sekretorische Funktion der Rinde und der Marksubstanz.

Ein unbefangener Blick auf einen Schnitt durch das Organ macht sofort den Eindruck, dass es sich um zwei strukturell und funktionell gänzlich verschiedene Abschnitte - Rinde und Mark handeln muss. Dieser Anschauung wird auch mit grosser Entschiedenheit von mehreren Autoren in ihren Abhandlungen über die Nebenniere Ausdruck gegeben. So sagt z. B. Swale Vincent: "These organs consist of two separate and distinct glands, cortex and medulla."

Guieysse kommt auf Grund seiner Untersuchung der Nebennieren des Meerschweinchens zum Ergebnis, dass nur der Rinde sekretorische Funktion zukommt, dass dagegen die funktionelle Bedeutung der Marksubstanz völlig dunkel bleibt. Er tritt mit grosser Entschiedenheit für die scharfe Sonderung der beiden Teile der Nebenniere ein. Die embryologische Erforschung der Nebennieren führte die meisten Untersucher $\mathrm{zu}$ einem ähnlichen Ergebnis. Manche Forscher auf diesem Gebiete vertreten den Standpunkt, dass an der Entstehung der Rinde epitheliale Zellen beteiligt seien, das Mark dagegen von sympathischen Zellen herrühre. Es wurden aber auch andere Ansichten über die Entstehung der Marksubstanz und ihr Verhalten zur Rinde geltend gemacht. So wurde schon von Gottschau die angebliche nervöse Herkunft der Markzellen geleugnet und die Möglichkeit eines Überganges der Rindenzellen in das Mark ernstlich in Betracht gezogen. Der neueste Autor auf diesem Gebiete, A. Roud (Contribution à l'étude du développement de la capsule surrénale de la souris) stellt ebenfalls eine zweifache Herkunft der Nebenniere in Abrede. - Er fand vielmehr eine indifferente, aus eigentümlichen Zellen bestehende embryonale Nebennierenanlage, in welcher eine nähere Differenzierung am 18. Tage beginnt. Die Differenzierungsvorgänge laufen gleichzeitig sowohl 
im Zentrum, wie an der Peripherie des Organes ab. Deutliche Übergänge zwischen Mark- und Rindenzellen finden zuweilen bis in sehr späte Stadien statt, ja kommen sogar beim erwachsenen Tiere vor. Bei solchen Widersprüchen ist es wohl zur Zeit nicht erlaubt, auf Grund der Entwicklungsgeschichte die Möglichkeit des Überganges der Rindenzellen in Markzellen direkt zu leugnen, unsere Präparate der Nebenniere der Maus sprechen jedoch in entschiedenster Weise gegen das Stattfinden eines solchen, wenigstens beim erwachsenen Tiere. Als Haupthindernis für einen Übergang der Rindenzellen in das Mark betrachte ich das dichte Gefässnetz, welches an der Grenze zwischen beiden Schichten verläuft. Am deutlichsten ist dieses Netz speziell bei den kleinen Nagern, der Maus und der Ratte zu sehen. Insbesondere bei diesen Tieren konnte ich nun zahlreiche Mitosen, nicht nur in der Rinde, wo sie bei sämtlichen Tieren zu finden sind, sondern auch im Mark (viel seltener) beobachten. Diese letztere Tatsache scheint mir von einer gewissen Beweiskraft gegen die Wahrscheinlichkeit des Überganges, bezw. der fortschreitenden Umwandlung von Rinde in Mark zu sein, da ja die etwa bei der Sekretionstätigkeit zugrunde gehenden Markzellen ihren Ersatz durch Teilung ihres gleichen und nicht durch Umwandlung nach innen gewanderter oder geschobener Rindenzellen zu bewerkstelligen scheinen. Ein weiterer Beweis der Unmöglichkeit eines Überganges der Rindenzellen in Markzellen in Verbindung mit einer direkten Migration von aussen nach innen, bieten die Verhältnisse der Zona reticularis, wie sie besonders deutlich beim Igel, aber auch bei der Ratte zum Ausdruck kommen. - Jede Zelle der Reticularis ist von dichtem Bindegewebe umsponnen, ja eigentlich in eine bindegewebige Scheide eingebettet, sodass ihre Lage dadurch als fixiert erscheint. Er würde auch der Habitus der Rinde-, resp. der Markzellen, namentlich der Grössenunterschied im Reichtum an Protoplasma sehr entschieden dagegen sprechen. Bei der starken Vakuolisierung des ganzen Zellleibes einerseits, wobei sogar der Kern durch riesige Vakuolen zur Seite geschoben wird (Rindenzellen), bei der kompakten Beschaffenheit des Protoplasmas andererseits (Markzellen) und bei dem Fehlen jeder Spur eines allmăhlichen Überganges ist jede Annahme der Umwandlung der einen Zellenart in die andere unzulässig. 
Unsere Untersuchungen führten uns ausserdem zu der Überzeugung, dass Rinde und Mark grundverschiedenen Funktionen obliegen, welche wohl beide für den Organismus gleich notwendig sein werden. Sowohl die Rinden- als auch die Markzellen können sich für sich vermehren, können für sich funktionieren, Zeichen von Erschöpfung ja sogar vom Absterben aufweisen. Für die Annahme einer Wanderung von der Peripherie zum Zentrum haben wir dagegen bis jetzt keine Anhaltspunkte gewonnen.

Der hier vertretene Standpunkt von der Spezifizitat von Mark und Rinde berührt allerdings nicht die Möglichkeit einer temporär auftretenden Umwandlung der beiden Zellarten in einander, die an besondere Zustände des Organismus, wie Schwangerschaft, Brunstzeit, verschiedene Ernährungszustände und dergl. geknüpft sein könnte. Unser Material bietet keine in diesem Sinne $z u$ deutenden Bilder bis auf ein Kaninchen, bei welchem in den Zellverband der Markzellen an der Peripherie des Markes hie und da typische Reticulariszellen eingeschoben sind. Die Deutung dieses Befundes bleibt unklar. Bezüglich des Überganges von der Rinde in das Mark wurde von mehreren Histologen, neuerdings von Flint, die Tatsache festgestellt, dass man in der Rinde zuweilen einzelne oder sogar ganze Gruppen von Markzellen trifft und auch umgekehrt, Rindenzellen im Mark. Diese Befunde kann ich ebenfalls bestatigen. Es ist aber nicht einzusehen, wieso diese Befunde für die Möglichkeit einer Umwandlung von Rinde in Mark verwertet werden dürfen.

Es ist darin wohl nur eine zufallige Verschleppung einer Zellengruppe zu erblicken, die wahrscheinlich noch in embryonaler Zeit, während des Vorganges der Anlagerung der Rinde an das Mark stattgefunden hat. Die vereinzelten Zellinseln können längere Zeit persistieren, weil es sich ja um ein noch zur Proliferation befähigtes Material handelt (Mitosen).

Es gelingt gar nicht selten in der Reticularis Zellen zu finden, welche auf den ersten Blick an Markzellen erinnern; ein näheres Eingehen auf ihre Struktur zeigt jedoch typische Abweichungen im Bau von den Markzellen, sodass ihre Spezifizität als Rindenzellen auch hier nicht in Frage gestellt werden kann (Kaninchen). Es bleibt nur noch übrig, zur weiteren Stütze des oben gesagten an die Beschaffenheit und Struktur der Nebennieren verschiedener niederer Wirbeltiere $z u$ erinnern. Die 
Suprarenalkörper der Teleostier und Ganoiden bestehen nur aus Rindenzellen, die Marksubstanz fehlt völlig.

Die Elasmobranchier besitzen zwar sowohl Mark als Rinde, beide Teile sind aber räumlich getrennt und liegen in einigem Abstande von einander. Die wirkliche genaue Verschiedenheit der beiden Abschnitte der Nebenniere ist somit wenigstens für einige Wirbeltiere direkt nachweisbar.

Es ist genugsam bekannt, dass das mikroskopische Bild der Rinde und des Markes ein sehr verschiedenes ist. Die Einteilung der Rinde in drei Zonen finde ich für sämtliche von mir untersuchte Tiere - Maus, Ratte, Meerschweinchen, Kaninchen, Igel, Mensch - gerechtfertigt. Die verschiedene Anordnung der Zellen in diesen drei Zonen wurde vielfach geschildert; ich möchte nur noch hervorheben, dass man bei einigen Tieren, namentlich der Katze, dicht unterhalb der Zona Glomerulosa eine Schicht stark vakuolisierter Zellen findet, welche Guieysse auch bei Meerschweinchen beobachtete und als "couche spongieuse" bezeichnete.

Die năhere Beschaffenheit der Zellen in den einzelnen Zonen kann folgendermassen kurz geschildert werden:

1) Zona glomerulosa.

Das Zellplasma ist nicht oder nur wenig kompakt, der Kern klein und zentral gelegen. Bei den meisten von mir untersuchten Tieren kommen in dieser Zone zahlreiche Mitosen vor. Die Form und Gruppierung der Zellen der Zona glomerulosa ist bei verschiedenen Tieren verschieden, so sind z. B. die Zellen der Glomerulosa des Hundes gewölbeartig peripher um meist radiäre Gefässe herum angeordnet; die einzelnen Zellen haben die Gestalt langer gedehnter Prismen; bei anderen Tieren sind die Zellen viel kürzer und breiter.

Die Zona fasciculata bietet ein etwas anderes Bild: die Zellen sind an Grösse denjenigen der Glomerulosa gleich, oder auch etwas grösser; sie zeigen im Gegensatz zu den Glomerulosazellen zahlreiche Vakuolen, welche an Menge von der Peripherie zum Zentrum hin zunehmen; der Kern ist durch andrängende Vakuolen aus seiner zentralen Lage verdrängt. Die Zellen in der Fasciculata haben annähernd prismatische ziemlich regelmässige Form, da sie in regelmässigen Reihen zwischen parallel verlaufenden 
Gefässen eingeschaltet sind. Die Fasciculata der Katze zeigt die vorhin bereits erwähnte eigentümliche Gliederung in eine mit stark vakuolisierten Zellen ausgestattete "Couche spongieuse" (Guieysse) und in eine tiefere Schicht mit mehr kompakten, auch bei den anderen Tieren wiederkehrenden Zellen. -

In besonders hohem Masse ist die Vakuolisierung der Rindenzellen beim Meerschweinchen ausgesprochen, wo Zellleib und Kern zuweilen völlig zurücktreten und durch eine kolossale Vakuole verdrängt werden. Solche Zellen werden sehr häufig und ganz unabhängig vom Geschlecht und physiologischen $\mathrm{Zu-}$ stande des Tieres vorgefunden. Beim Kaninchen scheinen dagegen ähnliche Vakuolen völlig zu fehlen; das Protoplasma bildet hier vielmehr, ähnlich wie auch bei der Ratte, ein feines, regelmässiges Wabenwerk.

Die Reticularis bietet hinsichtlich der einzelnen Zellen gegenüber der Fasciculata keinerlei Besonderheiten. Die Vakuolisierung der Zellen ist fast ebenso bedeutend, wie in der vorhergehenden Zone. Die Zellen liegen jedoch nicht mehr reihenförmig, sondern, indem sie sich dem durch die Gefässe gebildeten dichten Netze anpassen, sind sie gruppenweise in den Maschen des letzteren gelagert. An Grösse stehen die Reticulariszellen denjenigen der übrigen Rinde um ein bedeutendes nach. Eine Eigentümlichkeit in der Verteilung des Protoplasmas ist in den Reticulariszellen des Kaninchens anzutreffen: man kann öfters zwischen drei zusammenstossenden Zellen eine scharf begrenzte dreieckige Lücke oder zwischen zwei Zellen (Fig. 1) eine solche von mehr ovalem Querschnitt wahrnehmen, eine lücke, von der man nicht mit Sicherheit sagen kann, ob sie ein interzelluläres Kanälchen oder ein durch Schrumpfung entstandenes Kunstprodukt ist. Die bei EisenHamatoxylin schwarze Konturierung kann wohl durch Anhaufung von Farbe an den Wänden des engen Spaltes erzeugt werden. Die dem kleinen interzellulären Raume zugekehrten Plasmaabschnitte sind durch ihre Dichtigkeit und dunkle Färbung von dem übrigen, vakuolisierten Protoplasma deutlich unterschieden.

Eine sichere. Deutung dieses Bildes konnte vorlaufig nicht gegeben werden.

Die Gefässverhaltnisse der Rinde der Nebenniere wurden von den meisten Autoren in der Weise geschildert, dass von den an die Organoberfläche herantretenden Arterien sich 
reichliche Zweige senkrecht in das Innere der Rinde einsenken und in regelmässigem Radiärverlauf durch die Fasciculata ziehen. An der Grenze der Reticularis angelangt, bilden die Gefässe ein dichtes Netz, welches durch seine Maschen die eigentümliche Anordnung der Zellen verursacht. Die Gefassversorgung der Rinde scheint demnach vorwiegend arteriell zu sein, und zwar im Gegensatze zum Mark, welches ein reichliches venöses System bèsitzt.

Über die nähere Beschaffenheit der kleineren zwischen den Zellstrăngen der Fasciculata und Reticularis und den Zellgruppen der Glomerulosa gelegenen Gefässe, sowie über die Beziehungen zu den Zellen selbst sind uns nähere Angaben nicht bekannt. Ebenso wenig wurden irgend welche spezielle Abfuhrwege für die angeblich lebhaft sezernierenden Rindenzellen beschrieben. Ein nach dem gewöhnlichen Verfahren fixiertes und gefärbtes Prăparat ist auch gar nicht imstande, uns eine Vorstellung davon zu geben, wie intim die Beziehungen der Blutgefässe zu den Zellen der Rinde sind.

Es ist auch bis jetzt die wichtige Tatsache unberücksichtigt geblieben, dass wir es in den kleinsten Gefässen und Kapillaren der Rinde nicht mit völlig geschlossenen Wänden und nicht mit einer Zirkulation des Blutes bloss innerhalb derselben $\mathrm{zu}$ tun haben, sondern dass durch grössere Defekte in der Endothelialauskleidung ein Austreten des Blutes oder wenigstens des Plasmas aus den Gefässen in die peri- und interzellulären Rüume ermöglicht ist, kurz, dass die Zellen direkt vom arteriellen Blut umspült werden.

Diese eigentümlichen Beziehungen der Zellen zu dem Gefässsystem wurden von uns durch vitale Infusion von feinst zerriebener Tusche aufgedeckt.

Als Versuchstiere dienten uns Ratte, Kaninchen und Meerschweinchen; die besten Resultate erzielten wir bei der Ratte.

Das Tier wurde sehr tief atherisiert, - der Brustkorb schnell und ohne Blutverlust aufgeschnitten, das in schnellem Tempo (Ätherwirkung) schlagende Herz vorsichtig durch die Wunde herausgeholt, durch einen kleinen Stich in die linke Kammer wurde eine feine Kanüle in die Aorta eingeführt und um die Kanüle durch die Herzwand wurde eine Ligatur angelegt. Die ziemlich verdünnte Tusche-Kochsalzlösung wurde unter schwachem Druck - ca. $80-100 \mathrm{~cm}$ Druckhöhe der Lösung - 
iu rhythmischen, den Herzschlägen entsprechenden Absätzen in kleinen Mengen zu ca. 2-3 ccm eingeführt. Das Herz arbeitete weiter, die Tusche wurde somit gewissermassen auf natürlichem Wege mit dem Blutstrom den Organen zugeführt und konnte gleichmässig überall bis in die feinsten Gefässe gelangen. - Bei Anwendung der Infusion in die jugularis bleibt $\mathrm{zu}$ viel Tusche im kleinen Kreislauf an den Wänden der Gefässe abgesetzt. Um den Blutdruck durch die Einführung der ziemlich bedeutenden Mengen von Flüssigkeit (50 ccm, Ratte) nicht $\mathrm{zu}$ steigern, wurde eine kleine periphere Vene angeschnitten und durch sie liess man das Blut heraussickern.

Die Ergebnisse der Versuche waren nicht in allen Fällen die gleichen. Es gelangte die Tusche bald nur in die grösseren Gefässe, in vielen Fällen aber in die weiter unten zu beschreibenden interzellulären Răume; es ist ziemlich schwer, die richtige Konzentration der Tuschelösung herauszufinden, bei welcher das letztgenannte Resultat auftritt. Der Erfolg der Injektion konnte sofort an der Rinde der Nebenniere geprüft werden; war sie gelungen, so war das gelbe Organ gleichmässig grau verfärbt; in anderen Fällen waren nur die makroskopisch sichtbaren Gefässe schwarz. Es kommt auch häufig vor, dass die eine oder die andere Arterie durch Verstopfung an der Aufnahme der Tusche verhindert wird; der von ihr versorgte Rindenbezirk bleibt dementsprechend gelb. Um bei Herausnahme der Nebenniere Blut und Tuscheverluste und anfallige Verschiebungen der Tuscheablagerungen $\mathrm{zu}$ verhindern, tut man am besten, für kurze Zeit das ganze Tier in grosse Mengen der Fixierungstlüssigkeit einzulegen und erst nach ein paar Stunden, nach erfolgter Blutgerinnung die Nebennieren herauszuschneiden und weiter zu fixieren. Man kann aber auch alle grossen Gefässe unterbinden und das Organ sofort herausnehmen. Das Tier starb gewöhnlich noch während der Operation; es wurde andernfalls sofort nach der Operation getötet and jeweilen in Zenker'scher Flüssigkeit fixiert.

Das mikroskopische Bild (Färbung mit Hämalaun-Eosin) war nun in gelungenen Fällen folgendes (Fig. 2-3, Ratte): Die kleinen interzellulären Răume waren in allen Rindenbezirken mti Tuschepartikeln ausgefüllt. Die Zellen der Glomerulosa und Fasciculata waren sogar von einem fast vollständigen, scharfen, 
zierlichen schwarzen Kontur umgeben; die Tuschanhäufungen in der Reticularis, wo die Zellen kleiner, die interzellulären Räume grösser sind, waren noch mächtiger. Die Tusche drang aber nicht nur in die interzellulăren Räume, sondern sogar ins Innere der Zellen ein (Fig. 2-3). Ich konnte mich bei der Ratte mit grösster Deutlichkeit von der Anwesenheit feiner intrazellulärer, mit Tusche ausgefüllter Kanälchen überzeugen. Die Kanälchen zweigen sich von den intrazellulären Spalten $a b$ und heben sich mit grosser Schärfe vom hellrot gefärbten Hintergrund des Zellprotoplasmas ab. Ich konnte einige Male beobachten, wie ein intrazelluläres Kanälchen bis dicht an den Zellkern herantrat; in den meisten Fällen freilich wird in dem Schnitt nur ein Teil des Kanälchens getroffen. Man konnte sich stets überzeugen, dass die Kanälchen in keiner offenen Verbindung mit den zahlreichen Vakuolen stehen; nie habe ich die Tusche sich in dieselben ergiessen sehen, stets war der schwarze Inhalt der Kanälchen von einem deutlichen Saume des roten Protoplasma umgeben.

Ähnliche, wenn auch weniger deutliche Bilder nach vitaler Infusion konnte ich auch beim Kaninchen und Meerschweinchen auffinden. Beim ersteren konnte ich allerdings befriedigende Resultate nur mit wirklichen Farblösungen erzielen; die interzellulären Spalten scheinen hier zu fein zu sein, um den TuschepartikeIn Durchlass zu gewähren. Es muss zu diesen Versuchen mit Farbstoffen eine wässerige Farblösung gewăhlt werden, welche sich schnell und vollständig fällen lässt und bei guter Fixierung eine eventuelle Nachfärbung gestattet. Ich verwendete Toluidinblau, Fällung mit Sublimat, nachträgliche Molybdänierung (siehe Gurwitsch: zur Morphologie und Physiologie der Nierentätigkeit). Die Fällung der Farbe konnte nun an den oberflăchlichen Partien des Organes schnell genug vor sich gehen; es waren somit nur die Zona Glomerulosa und Fasciculata zu brauchen, in den tieferen Partien war das Bild durch starke Diffusion der Farbe und eine wirkliche Färbung der Zellen stark getrübt.

In den gut fixierten Partien konnte man die interzellulären Spalten als feine, scharf gezeichnete, aus kleinsten Förnchen von gefälltem Toluidinblau bestehende Linien erkennen. Die Zellkonturen waren durch diese Linien vollständig und allseitig umzeichnet. Dass es sich um tatsächliche Fällung von frei zirkulierendem Farbstoff und nicht um wirkliche Färbung der 
Zellgrenzen (Kittsubstanz) handelt, ergibt sich: 1. aus der Farblosigkeit des Zellplasmas und der Zellkerne, 2. aus der Fällung des Farbstoffes in Form von Körnchen, was bei Fixierungen einer echten Färbung mit Toluidinblau nie der Fall ist (wie sich aus anderen Stellen des Präparates nachweisen lässt). Ganz ăhnliche Bilder wurden neuerdings von Ciaccio unter Anwendung der Golgi'schen Methode für endozelluläre Netze beschrieben. Den Beobachtungen von Ciaccio könnte allerdings der Einwand gemacht werden, dass durch Reduktionsbilder des $\mathrm{Ag} \mathrm{NO}^{3}$ die Natur der Streifen als wirklicher Kanäle durchaus nicht bewiesen erscheint, da ja auch Kittsubstanzen usw. das $\mathrm{Ag} \mathrm{NO}^{3}$ zu reduzieren vermögen, wie ja auch das Wesen des "Apparato reticolare" noch durchaus dunkel ist. Unsere Bilder vermögen dagegen für die Deutung derjenigen von Ciaccio eine Stütze zu bieten und ergänzen sich mit denselben gegenseitig.

Es wurde bereits oben hervorgehoben, dass die Rinde ausschliesslich von arteriellem Blute versorgt wird; die intrazellulären Blutwege, welche durch unsere Injektionen nachgewiesen wurden, führen somit arterielles Blut oder wenigstens Blutplasma nicht nur bis an die Zelloberflache heran, sondern vermöge der intrazellulären Kanäle sogar in das Zellinnere hinein; die Spalten sind allerdings meistens zu klein, um auch den Blutkörperchen den Durchtritt zu gewähren, das Blutplasma aber kann ungehindert ins Innere der Zellen und in ausserordentlich tiefgehende Berührung mit dem Zellleib gelangen.

Diese so merkwürdig reiche Blutversorgung der Rindenzellen muss meines Erachtens, abgesehen von der Bedeutung für die Zellernährung, auch in einer anderen speziellen Funktion der Rindenzellen ihren Grund haben. Es wird im Folgenden wahrscheinlich gemacht werden, dass, entgegen der vielfach geltenden Ansicht diese Funktion nicht in einer Sekretion von den Zellen in das Blut hinein, sondern umgekehrt in einerStoffaufnahme aus dem Bluteseitens derZellen, und zwar höchstwahrscheinlich in einer Entgiftung des Blutes besteht.

Ich stelle mich mit dieser Behauptung in einen Gegensatz zu mehreren Autoren, u. A. zu Guieysse, welcher den Standpunkt vertritt, dass der Rinde ausgesprochene sekretorische Funktionen zukommen. Er stützt sich auf eine Reihe von Be- 
obachtungen an trächtigen und nicht trächtigen Meerschweinchen, und auf ihr Verhalten im Vergleich zu den Männchen. Guieysse glaubt in der Zunahme der Vakuolisierung der Rindenzellen während der Schwangerschaft den Beweis für ihre sekretorische Funktion erblicken zu müssen. Der spongiösen Schicht soll seiner Ansicht nach die Ausarbeitung der flüssigen Bestandteile des Sekretes, vielleicht nur des $\mathrm{H}_{2} \mathrm{O}$ und der Salze zufallen, die Zona fasciculata dagegen liefere die übrigen, festeren Bestandteile des Sekretes, welche sich in dem von der vorhergehenden Schicht gebildeten Sekret lösen sollen. Die dritte Schicht, die Reticularis, soll sich durch ihren Pigmentgehalt und die "corps sidérophiles" auszeichnen. Die ganze Menge des Sekretes soll sich nun in die venösen, in der Marksubstanz gelegenen Sinus ergiessen. Es bleibt in Guieysse's Beschreibung völlig unaufgeklärt, welchen Weg die durch die verschiedenen Schichten erzeugten Sekretbestandteile einschlagen. Gelangt das Sekret direkt ins Blut, oder dringen die Produkte der distal gelegenen Zellen auf anderem Wege in die mehr zentral gelagerten Regionen? Es fehlt bei Guieysse jeder Nachweis von speziellen Ausführungsgängen, die ja meines Erachtens notwendig vorhanden sein müssten, wenn man die kolossalen Sekretmengen in Betracht zieht, die nach Guieysse's Ansicht von den Zellen, die von Sekretvakuolen strotzen, geliefert werden müssen.

Ich konnte aber trotz aufmerksamen Studiums meiner Präparate bei verschiedenen Tieren und verschiedenen Färbemethoden keine Spur eines ableitenden Kanalsystems entdecken. Der andere für die Entleerung des Sekretes noch mögliche Weg — seine Ausstossung direkt in das Blut ist in diesem Fall kaum denkbar, da die vorliegenden Bedingungen sehr ungünstig zu sein scheinen. Die Zellen kommen nicht mit venösem, sondern direkt mit arteriellem unter relativ hohem Druck stehendem Blut in Berührung. Dass die anzunehmende, das Sekret ausstossende Kraft dem Blutdrucke nicht gewachsen ist, erhellt schon daraus, dass die im Blut suspendierten Tuschpartikeln so leicht bis in die feinsten Interstitien, ja sogar bis in die Zellen hinein vordringen; es kann somit kein gegen die Richtung des Blutdruckes gerichteter Strom in den interzellulären Răumen oder intrazellulären Kanälen während des Lebens vorhanden sein. 
Eine korpuskuläre Ausstossung von Sekret erscheint auch nach dem mikroskopischen Befunde der grossen Zellen sehr unwahrscheinlich; die Vakuolen sind von der Aussenfläche der Zellen stets durch eine deutliche, ununterbrochene Plasmalage geschieden; auch die intrazellulären, mit Tusche gefüllten Kanäle kommen nirgendswo mit einer Vakuole oder ihrem Inhalt in Berührung, sind vielmehr stets von denselben deutlich geschieden. Wir sehen somit, dass die Rindenzellen nicht für sezernierende Zellen angesprochen werden dürfen, sondern dass ihnen mit viel grösserer Wahrscheinlichkeit eine entgegengesetzte Funktion, diejenige der Stoffentnahme aus dem Blute zugeschrieben werden muss, wozu sie sich namentlich auch durch ihre Beziehungen zum Blutgefässsystem ganz besonders eignen. Die Entgiftungs-Arbeit der Zellen der ihrem Volum nach so geringen Nebenniere muss allerdings sehr bedeutend sein.

Wir wollen nun versuchen, unsere Hypothese näher zu begründen und die zu Gunsten derselben sprechenden Gründe anzuführen. Von Wichtigkeit ist für uns vor allem der chemische Charakter der Zelleneinschlüsse der Rindenzellen, d. h. ihrer zahlreichen Vakuolen. Alexander hat den Nachweis erbracht, dass die Nebenniere in ihrem Lecithinreichtum nur dem Gehirn nachsteht. Durch die Befunde von zahlreichen Autoren wissen wir, dass die Vakuolen fettartige Substanzen einschliessen. M u lon gibt neuerdings direkt an, es handle sich um Lecithin. Wie lassen sich nun diese reichen Lecithinanhäufungen erklären?

Zur Erklärung dieses auffallenden Lecithingehaltes stellt Alexander folgende Hypothese auf: Die Beziehungen der Erkrankungen des Zentralnervensystems zu denjenigen der Nebenniere, z. B. der Addison'schen Krankheit, welche mit einem Komplex von nervösen Störungen einhergeht, und ebenso auch die Beziehungen der Nebennierenaplasie zur Gehirnaplasie führen unwillkürlich auf den Gedanken, dass die Nebennierenzellen und die Gehirnzellen, welche beide durch den reichsten Lecithingehalt ausgezeichnet sind, in engsten Beziehungen zu einander, wenigstens in der Entwicklungsperiode stehen. Alexander vermutet nun, dass die Nebenniere als Vorratskammer und als Fabrik für das Gehirn dient und das für letzteres Organ notwendige Lecithin liefert.

Diese Aufstellung Alexanders kann nur den Wert einer Hypothese beanspruchen und stösst auf manche Schwierigkeit; 
der Gedanke verdient aber jedenfalls alle Beachtung und die Nachprüfung der Frage, inwiefern das in den Vakuolen der Rindenzellen so reichlich aufgespeicherte Lecithin von denselben ausgeschieden oder sonstwie aufgebraucht wird, ist dringend geboten. Erst nach Aufklärung dieser Frage wird ein bestimmtes Urteil sowohl über die Richtigkeit der Hypothese von Al ex and er, als auch über die Funktion der Nebenniere möglich sein. ${ }^{1}$ )

Ich habe bereits oben erwähnt, dass bezüglich der Funktion der Rindenzellen mehr Anhaltspunkte für folgende Hypothese vorhanden sind: Die Rindenzellen sind in einer ganz besonderen Weise zur Aufnahme von verschiedenen, im Blute gelösten Stoffen, vielleicht giftigen Stoffwechselprodukten, somit zur Entgiftung des Blutes adaptiert, aussert sich doch auch der Ausfall der Nebennierentätigkeit in einer Autointoxikation des Organismus! Es wurde bereits oben der ausserordentlich intimen Beziebungen der Zellen zum Blut, und zwar vorwiegend zum arteriellen Blute Erwähnung getan. Der ausserordentliche Lecithingehalt der Rindenzellen, wobei das Lecithin im Gegensatze zu allen übrigen Körperzellen (auch zu den Markzellen) nicht chemisch gebunden zu sein scheint, führt uns auf die namentlich durch die Untersuchungen von $\mathrm{O}$ verton zu Tage getretene Wichtigkeit desselben für die Aufnahme der Stoffe von aussen. - Overton gelangte, wie bekannt, auf Grund ausgedehnter Untersuchungen zum Schlusse, dass nur diejenigen im umgebenden Medium zirkulierenden Stoffe im allgemeinen durch lebende Zellen aufgenommen werden, welche mehr oder weniger in lipoiden Stoffen (Lecithin, Cerebrin, Cholestearin, Protagon) löslich sind. Man muss somit annehmen, dass die Plasmahaut der Zellen einen lipoiden Überzug besitzt. oder von Lipoiden imprägniert ist.

Wenn somit der Lipoidgehalt der Plasmahaut für die Durchlässigkeit massgebend ist, so liefern die lecithinreichen Granula der Rindenzellen den letztern die Möglichkeit, sämtliche in die Zellen gelangten Stoffe in höherem oder geringerem Masse aufzunehmen und aufzuspeichern. Von dem sogenannten Teilungs-

1) Es sei noch hervorgehoben, dass nach A le x a n d e r \& Angaben der Lecithingehalt der Markzellen der Nebennieren noch reichlicher ist, wie derjenige der Rindenzellen. Das Lecithin scheint allerdings in letzteren Zellen chemisch gebunden, jedenfalls nicht abgespalten zu sein; es lässt sich weder morphologisch noch mikrochemisch nachweisen. 
coefficienten der Löslichkeit der betreffenden Stoffe im Blutplasma, resp. im Lecithin, wird es natürlich abhängen, ob sie in den Rindenvakuolen in grösserer oder geringerer Konzentration aufgestapelt werden. - Wenn wir nun die Annahme machen, dass einige im Blutplasma gelöste toxische Stoffwechselprodukte, die bei Addison'scher Krankheit die Autointoxikation des Organismus verursachen, eine starke Löslichkeit in Lecithin aufweisen, so genügt dies, um ihre Aufspeicherung in der Nebennierenrinde und die Entgiftungsfunktion der letzteren zu erklären. - Dass diese Annahme an und für sich sehr zulässig ist, erhellt bẹi Berücksichtigung der Lösungsfähigkeit der Lipoide für viele Stofte (Overton).

Die Rinde der Nebenniere ist somit unserer Ansicht nach kein sezernierendes Organ, sondern ein umgekehrt funktionierendes - ein Aufnahmeorgan. - Dass die Rinde in ihrem Bau keine Charaktere einer Drüse enthält, ergibt sich aus unserer mikroskopischen Schilderung. Es ist aber andererseits leicht einzusehen, wie sehr die eigentümlichen Beziehungen der Rindenzellen zum arteriellen Blutgefässsystem geeignet sind, die von uns angenommene Funktion zu fördern. Was das weitere Schicksal der im Lecithin aufgespeicherten toxischen Stoffwechselprodukte betrifft, so kann man sich denken, dass sie einer Zersetzung, vielleicht einer Oxydation anheimfallen und dass die dabei entstehenden niedrigmoleculären Zerfallsprodukte aus den Zellen ins Blut diffundieren können.

Wenn wir auf die feineren Verhältnisse im Baue der Rindengefässe eingehen, welche den Eintritt der corpuscularen Elemente, somit auch des Blutplasma nicht nur in die feinsten interzellulären Spalten, sondern auch in die Zellen hinein ermöglichen, so muss zunächst festgestellt werden, dass es sich keinesfalls um feinste, von besonderer Membran oder Kruste geschlossene Röhrchen, etwa vergleichbar den Gallenkapillaren, handeln kann; eine Wandung ist nicht $\mathrm{zu}$ sehen; ebenso wenig findet man rundliche Querschnitte, wie sie bei röhrenförmigen Kanalchen notwendigerweise hie und da vorkommen müssten und ja auch tatsăchlich bei Gallenkapillaren und interzellularen Drüsengängen sehr deutlich sichtbar sind; in unseren Tuschepräparaten müsste es sich um scharf umschriebene, rundliche Anhäufungen von Tusche handeln. Man sieht aber nur feine schwarze Linien, die gewöhnlich die 
Zelle ringsherum umgeben und somit optische Schnitte durch Spalten sein müssen. Als einzige Errklärung bleibt die Annahme, dass die Wände der kleineren Gefässe, resp. der Kapillaren, nicht wie in den sonstigen Organen abgeschlossene Röhren darstellen, sondern an vielen Stellen Lücken besitzen.

Es ist mir auch ein direkter Nachweis dieses Verhaltens an Kapillarwänden gelungen.

. Wie wir aus den Figuren 2 und 3 ersehen, erscheint hier das Lumen eines mittleren Kapillargefässes zum grössten Teil von jeder endothelialen Auskleidung entblösst; für die ganze Peripherie des Gefässes (Fig. 3) sind zwei sehr scharf gezeichnete und nach allen Seiten abgegrenzte Endothelzellen mit Kern sichtbar, welche nur einen Teil der anliegenden Fasciculatazellen zudecken und gegen den Blutstrom abgrenzen. Das Bild erinnert in gewisser Hinsicht an die durch von $\mathrm{Kupffer}$ für die Blutkapillaren der Leber gegebene Schilderung. Seine Figuren zeigen in einer ganz ausgesprochenen Weise auffällig weite Abstände der Endothelzellen, deren lange protoplasmatische AusIïufer untereinander netzartige Anastomosen bilden. Durch die so gebildeten Lücken zwischen den Endothelzellen kann wohl das Blutplasma und können unter Umständen auch Blutkörperchen eindringen. An unserer Abbildung No. 3 können wir uns nun überzeugen, dass an der vom Lumen abgewendeten Seite der Endothelzellen, zwischen ihrien und den Fasciculatazellen Tuschpartikel liegen. Demnach ist der Tusche resp. dem Blutplasma freier Zutritt zu den interzellulären Spalträumen gestattet. Ich glaube, dass die einzige Möglichkeit zum Austritt der Tusche aus der Blutbahn eben in der eigentümlichen lückenbaften Beschaffenheit der Gefässwand zu suchen ist, wie v. Kupffer sie für die Leber beschrieben hat und ich sie für die Rinde der Nebenniere nachweisen kann. Von Gefässrupturen kann bei meinem Verfahren keine Rede sein, da ja die Injektion keine forcierte war und ausserdem durch öffnen einer Vene für freien Abfluss des Blutes gesorgt wurde. Abgesehen von der Lückenhaftigkeit des Endothelbelages sind freilich die Unterschiede der Kapillarwand der Leber und der Nebenniere sehr bedeutend, infolge der eigentümlichen Verhältnisse der Sternzellen der Leber. Letzteren kommt nach von $\mathrm{Kupffer}$ 's Untersuchungen eine sehr hochgradige Fähigkeit der Phagocytose zu. Sie verschlingen 
nicht nur Erytrocyten, sondern auch massenhaft Tuschpartikel, sie besitzen lange, zum Teil wohl amöboide Fortsätze. Eine ăhnliche Funktion ist nun offenbar beim Gefässendothel der Rindengefässe der Nebenniere nicht vorhanden; die Endothelzellen besitzen hier keinerlei protoplasmatische Fortsătze mit Anastomosenbildung. Ich kann mit Sicherheit behaupten, dass die Lücken der Gefässauskleidung der Kapillaren der Nebennierenrinde durch Ausfall oder Fehlen ganzer Endothelzellen bedingt werden und den Austritt der gelösten und ungelösten Stoffe aus der Blutbahn ermöglichen.

Die Wichtigkeit der intrazellulären Kanäle für die Ernährung der Zellen und namentlich für die von uns angenommene Funktion ist ohne weiteres ersichtlich, da ja eine möglichst grosse Oberfläche die Aufnahme von Stoffen durch die Plasmahaut fördert.

Die in vielen Zellen durch die Arbeiten von Holmgren, S tudnička u. A. beschriebenen Saftkanălchen werden gewöhnlich als ableitende Lymphwege angesehen. - Ein Beispiel von zuführenden Kanälchen ist durch die Injektionen von $\mathrm{S} \mathrm{c} \mathrm{h} \mathrm{a} \mathrm{f} \mathrm{fer}$ B rowic z u. A. in den Leberzellen bekannt geworden, in welchen, man wirkliche intrazelluläre, von der Arterie aus injizierbare Blutbahnen. nachweisen konnte ${ }^{1}$ ). Es ist im allgemeinen anzunehmen, dass für die Zuführung von Nahrstoffen zu den Zellen viel speziellere und intimere Beziehungen zur Blutbahn notwendig sind, als für die Abfuhr von Elaboraten der Zellen, schon aus dem Grunde, weil die Stoffwechselprodukte, welche die Zellen verlassen, meistens relativ niedrigmolecular und somit leichter diffusibel sind, als die den Zellen zugeführten Stoffe. Bei unserer Annahme einer entgiftenden Funktion der Rinde der Nebenniere und der Zersetzung der von den Rindenzellen aufgenommenen toxischen Stoffwechselprodukte innerhalb der Zellen wird deshalb die weitere Annahme als sehr naheliegend erscheinen, dass, wie wir bereits nachzuweisen versuchten, die inter- und intrazellularen Wege der Nebennierenrinde tatsacblich zuleitend sind. Wie wir gesehen haben, dringen die intrazellulären Kanäle tief in den Zellleib ein, um daselbst blind $z u$ endigen. Es kann somit von einem zuführenden

1) Die Angaben von $\mathrm{Browicz}$ wurden übrigens von $0 \mathrm{ppel}$ und $\mathrm{H}$ o I m g r e $\mathrm{n}$ in sehr abfälliger Weise beurteilt. 
Strom innerhalb derselben nur soweit die Rede sein, als deren Inhalt vom Zellleibe resorbiert wird. Um uns das leichte Eindringen der Tusche in dieselben zu erklären, müssen wir an eine, wenn auch geringe, durch den Resorptionsakt erzeugte Saugwirkung denken. Inwiefern das eben geschilderte Kanălchensystem auch für den diffusionellen Austritt von Stoffen aus den Zellen dienen kann, lässt sich aus naheliegenden Gründen nicht ermitteln; es sei nur hervorgehoben, dass jede Zelle mit irgend einer Seite einem in grösserer Ausdehnung wandlosen Gefässlumen anliegt.

Zur Vervollständigung des Bildes der Nebennierenrinde bleibt noch die Schilderung des bindegewebigen Stützgerüstes derselben zu geben. Die Bindegewebszüge folgen im allgemeinen den grösseren, „radiär" verlaufenden Gefässen und schicken feine Ausläufer zwischen die Zellgruppen und sogar zwischen die einzelnen Zellen des Parenchyms hinein.

Einzelne Fasern gehen zuweilen in transversaler Richtung ab. In der Reticularis, in welcher die Gefässe netzartig angeordnet sind, wird ebenfalls von Bindegewebszügen ein Reticulum gebildet, welches, von der Adventitia eines Gefassstammes ausgehend, radiär nach allen Seiten vordringt und die Reticulariszellen umspinnt; besonders deutlich habe ich diese Bilder beim Meerschweinchen gesehen. Die arteriellen Blutgefüsse der Rinde, welche in der Reticularis ein Netz bilden, ergiessen nun, in das Mark angelangt, ihr Blut in die weiten venösen Stämme und Sinuse, welche der Marksubstanz der Nebenniere ein eigentümliches Gepräge verleihen.

Die Marksubstanz funktioniert wie eine echte Drüse, in ihr sind nun wirklich alle die zur Sekretion nötigen Einrichtungen und Strukturen gegeben, welche der Rinde fehlen. Die allgemeinen Bauverhăltnisse der Marksubstanz sind, so wie ich sie aus meinen Präparaten habe erschliessen können, bereits in einer vorlăufigen Mitteilung (Anatomischer Anzeiger 1902) von mir dargelegt und auch in der vorliegenden Arbeit von neuem zur Sprache gebracht worden. Die Anordnung der Zellen zu charakteristischen Markläppchen ist prinzipiell die gleiche bei allen von mir untersuchten Tieren, wie Feldmaus, Maus, Igel, Ratte, Meerschweinchen, Kaninchen, Rind, Katze und Kalb, sowie beim Menschen. Die Plasmastrukturen der Zellen sind dagegen sehr 
verschieden, je nach der Tierspezies. Besonders auffallend ist die Beschaffenheit der Markzellen beim Igel, wo neben dem grossen Kern eine ebenso grosse Sphäre mit einem deutlichen Diplosoma sich von dem sehr lockeren, kaum färbbaren Cytoplasma abhebt. Beim Meerschweinchen haben die Markzellen ein ebenfalls ziemlich lockeres Gefüge, bei den übrigen Tieren ist die Struktur annähernd unter sich gleich, das Cytoplasma dichter, der Kern kleiner; von einer Sphäre ist nichts zu sehen.

Die architektonische Anordnung der Markzellen wird vollständig durch die Gefässverhaltnisse des Markes beherrscht. Wenn wir ein Übersichtsbild der Marksubstanz eines Kaninchens (Fig. 4), welches wir unserer Schilderung zu Grunde legen wollen, betrachten, so fallen vor allem die zahlreichen Durchschnitte durch weite, dünnwandige venöse Gefässe auf. Das zur Venenwand gehörende Bindegewebe scheint im Anschluss an die Venen mitunter septenartig sich auszubreiten. Die Maschen des sehr weiten, durch die Venen und allfallige anschliessende Septa gebildeten Netzes sind durch Markzellen ausgefüllt. Auf diese Weise bilden die Markzellen miteinander gewissermassen ebenfalls ein Balkenwerk, welches mit dem Netzwerk der Gefässe (und Septa) alterniert. Es ist dabei natürlich nicht ausgeschlossen, dass die einzelnen Markbalken stellenweise plattenartig verbreitert sein könnten, ăhnlich, wie solches bei den Leberzellbalken der Fall ist. In den Markbalken herrscht nun eine ganz eigentümliche Gesetzmảssigkeit in der Anordnung der Zellen. Die Zellen bilden in epithelähnlicher Anordnung eine geschlossene Aussenschicht um einen mittleren lacunären Raum. Die Kerne der Zellen finden sich in den innern, der Lakune zugekehrten Enden der Zellen eingelagert, während die kernfreien, fast homogen erscheinenden Aussenzonen der Markzellen die Oberfläche des Markbalkens einnehmen und vorzugsweise den grossen Venen und anschliessenden Septa zugewendet sind. Die lakunären Binnenräume oder Gangsysteme verlaufen nach dem Ausgeführten im Innern der Markbalken und verzweigen sich mit denselben, wobei es auch zur Anastomosenbildung zwischen denselben kommt, doch scheint es andererseits, dass in den Aussenbezirken des Markes, an der Grenze zwischen Mark und Rinde, die Lakunen blind endigen. Ist ein Markbalken der Länge nach getroffen, so sind die Markzellen um die Lakune palissadenartig angeordnet 
und gleichzeitig ist ihre radiäre Anordnung zur anliegenden Vene deutlich hervortretend. Im Querschnitt durch einen Balken ist die zelluläre Begrenzung des lakunären Raumes einem ganz oder fast ganz zum Ring geschlossenen Gewölbe zu vergleichen. Während im allgemeinen diese lakunären Binnenräume überall durch eine geschlossene Schicht von Markzellen von den Venen (und den ihnen sich anschliessenden Septen) getrennt sind, wird diese zellige Einfassung ab und zu durch eine Lücke unterbrochen. Durch diese Pforte tritt dann das Bindegewebe des lakunären. Spaltes mit den grossen Venen, resp. ihrer bindegewebigen Umgebung in Verbindung, und das kleine lakunäre Gefäss mündet durch diese Lücke in die Vene (Fig. $4^{*}$ ).

Im allgemeinen sieht man die Balken des Markes zwischen benachbarten Venen hindurch miteinander zusammenhängen, ebenso wie ihre zentralen Lakunen (letztere brauchen auf dem Schnitt nicht notwendig mitgetroffen zu sein). Mitunter aber sieht man zwischen zwei Venen zwei Markbalken mit ibren Aussenzonen aneinander grenzen. Zwischen denselben findet sich dann eine: enge Spalte, in welche das die Venen begrenzende Bindegewebe septenartig eindringt oder auch eine rinnenartige Ausbuchtung eines Sinus zur Ausfüllung eines grösseren Zwischenraumes, sich hineinzieht (Fig. 4). Überhaupt zeicbnen sich die Venen, welche den von den Markbalken frei gelassenen Raum ausfüllen, durch ihre unregelmässige Gestalt aus, entsprechend ihrem Verhalten als Gebilde, welche die Schalträume ausfüllen zwischen mehr oder weniger zylindrischen Markbalken, die sich beliebig verzweigen. Die Markbalken scheinen, den tubulo-alveolären Drüsen analog, im grossen und ganzen ihre zylindrische Form beizuhalten, sowohl den umgebenden Venen als der Rinde gegenüber, und in diese sowohl der Länge nach in ihrem Verlauf, als auch stellenweise mit längeren oder kürzeren buckelartigen freien Seitensprossen sich hineinzuwölben. Die venösen Sinuse dringen ibrerseits, wie schon gesagt, vielfach mit spitzwinkligen Buchten und Rinnen zwischen die Markzylinder ein.

Grössere flăchenhafte Ausbreitung der Markbalken und spaltartige Ausbreitung der im Innern gelegenen Lakune scheint im allgemeinen nicht vorzukommen; es müsste dabei ja doch ab und zu eine solche ausgebreitete Spalte flach getroffen sein, während man solche Bilder vermisst. 
Ein Querschnitt durch einen Markbalken in seinem mittleren Verlauf oder ein beliebiger Schnitt durch ein blindes, gegen die Rinde anstossendes Ende bietet uns das Bild, welches wir in unserer vorläufigen Mitteilung (Anatomischer Anzeiger 1902) mit dem Namen der "Markläppchen" belegt haben; wir werden statt dessen im weiteren nun auch die Bezeichnung "Markzylinder" oder "Markbalken" gebrauchen, indem wir von den vorkommenden buckel- oder gewölbeartigen Vorsprüngen in ihrem Verlauf absehen.

Das eigentümliche, im Innern der Markbalken gelegene, oben erwähnte Gangsystem der Lakunen ist besonders deutlich bei einigen Nagern (Meerschweinchen, Ratte und namentlich Kaninchen) ausgesprochen; bei den andern von mir untersuchten Tieren lăsst sich die Architektur der Balken und der Zusammenhang ihrer einzelnen Teile nicht so leicht erfassen.

Wie sich aus der vorhergehenden Schilderung ergibt, ist die Lakune keinesfalls als ein wirkliches wandloses Gefäss, eine Vene zu erklären, wie es M a n as s e u. A. getan haben, wenigstens nicht beim Kaninchen; die Lappchenzellen sind nicht die Wand der Blutgefässe, ihre Zellen sind auch nicht als eigentümliche Unterbrechungen in die Gefasswand eingeschaltet, stellen vielmehr neben oder $z$ wischen den Blutgefässen gelegene Gebilde dar, welche allerdings funktionell in einer ganz eigentümlichen Weise mit den Gefässen in Verbindung treten. Wenn man in der Mehrzahl der Falle auf einem Quer- oder Schiefschnitt durch den Markzylinder auch Querschnitte der Gefasse an den oben erwähnten Stellen der "Läppchen" findet, so sieht man andererseits auch häufig genug Bilder, welche auf eine eigentümliche seitliche Abknospung des längsverlaufenden Gefässes hindeuten. Die Figuren 8, 8a und 9, welche benachbarten Serienschnitten entnommen sind, zeigen, wie die Wand eines quer getroffenen Gefässes einen seitlichen Auslaufer aussendet, der in den Hilus eines Markbalkens eindringt. Die aus Endothel mit bindegewebiger Bekleidung bestehende Wand des Gefässes verdünnt sich und wird an einer Stelle gänzlich durchbrochen (Fig. 8, 8a u. 9) Im Lumen findet sich auf dem in Fig. 8 abgebildeten Schnitte ein Erythrocyt; auf dem nachsten Schnitt (Fig. 9) ist das nicht völlig ringsherum geschlossene Lumen des Ausläufer-Gefässes lănglich; das Klaffen desselben und eine gewisse Auffaserung etwa ähnlich dem von Weidenreich geschilderten bei den) 
Milzkapillaren) ist hier noch deutlicher. In Fig. 8a mündet schliesslich der Seitenspross in das Hauptgefäss. Es kann demnach keinem Zweifel unterliegen, dass eigentümliche Gefässsprossen frei in die Lakunen münden oder aus denselben entspringen. Die feinen Ausläufer der Gefảsswände treten häufig direkt an die Zellen heran, sodass ein zuweilen recht enger Spalt die Kommunikation zwischen Lakune und Gefässlumen vermittelt (Fig. 10). Wie bereits erwähnt sind die mit Lücken versehenen Gefässsprossen oft auf längere Strecken in der Längsachse eines Markzylinders zu verfolgen, bis sie sich dann schliesslich ebenfalls in der eben angegebenen Weise auflösen. Trifft man sie auf einem Längs- oder Querschnitte, so ist ihre Mündung (resp. ihr Ursprung) an den grösseren, dünnwandigen, venösen, intralobulären Gefässen sehr leicht nachweisbar. - Wir sehen somit, dass die Verbindung der geschlossenen Blutbahn mit dem Lakunensystem durch offene Seitensprossen des ersteren und nicht durch eine wirkliche Unterbrechung bewerkstelligt wird. $O b$ man nun die eigentümlichen offenen Gefässsprossen für Blutgefässe oder Lymphgefüsse ansehen soll, ist schwer aus den Präparaten zu entscheiden; an Inhalt weisen sie, wenigstens nach dem Tode, sowohl Erythrocyten wie auch relativ zahlreiche Leukocyten auf (Fig. 8, 9). Die stofflichen Verhältnisse des Lakuneninhaltes, welche für den Charakter der Gefässsprossen als bestimmend angesehen werden müssen, werden unten erörtert werden.

Eine Lagerung der Zellen um einen grösseren lakunenartigen Raum herum wurde in dieser Form nur einmal, bei Carlier erwähnt, welcher die Lakune für einen venösen Sinus erklärt und die Aussenseite der Läppchen von einem arteriellen Netz umsponnen sein lässt. Wir vermissen jedoch bei Carlier jede Andeutung über ein innerhalb der Lakune verlaufendes oder in dieselbe mündendes Gefäss, was natürlich für die Deutung der Verhältnisse massgebend ist. Das Fehlen desselben hat wohl auch Carlier zur Annahme bewogen, die Lakunen für einen venösen Sinus $z u$ erklären, während wir dagegen in den Lakunen eigentümliche perivaskulare Raume erblicken. Unsere Befunde an der Marksubstanz der $R$ at t e schliessen sich der Carlier'schen Schilderung besser an, indem wir hier in den Lakunen die einmündenden Blutgefässe vermissen, hier somit ihrer Deutung als venöser, wandungsloser Sinuse an und für sich nichts im Wege steht (Fig. 6). 
Was Carlier's Angaben über die angeblich arterielle Natur der an der konvexen Seite der Läppchen verlaufenden Gefässe betrifft, so glauben wir nicht, dass man diese Angabe mit voller Entschiedenheit aufrecht erhalten kann; wenn auch die relativ dicken Wände und das enge Lumen der betreffenden Gefässe namentlich bei der Ratte (Fig. 6) für ihre arterielle Natur sprechen, so sieht man andererseits ab und zu ihr direktes Einmünden in weite dünnwandige. Gefässe, die hie und da zwischen den Läppchen verlaufen und zweifellos Venen sind. Die Dicke der Wand kann natürlich nicht für die Natur der Gefässe massgebend - sein, handelt es sich doch nicht um Muskelzellenbekleidung, sondern nur um bindegewebige Elemente. Die grösseren, zwischen den Läppchen verlaufenden, vorhin erwähnten Venen bieten einige Eigentümlichkeiten; die Wand besteht einzig und allein aus Endothel, mit spärlichem, unterliegendem Bindegewebe, welches gleichzeitig die Kapsel der Läppchen bildet. Es sind aber auch unzweifelhafte, von mehreren Autoren bereits geschilderte Defekte in den Gefässwandungen vorhanden, wodurch es zustande kommt, dass stellenweise nackte Markzellen direkt aussen von venösem Blut bespült werden (Fig. 10); einzelne Zellgruppen können auch hie und da in das Innere des Gefässlumens etwas vorragen. Letzterer Umstand gab, wie bekannt, die Veranlassung zur Annahme, dass Zellgruppen von Venenstrom mitgerissen und weggeschwemmt werden, resp. dass protoplasmatische Zellteile in die Venen abgestossen werden (Got tscha u). Dass dies als physiologischer Akt nicht vorkommt, lässt sich an gut konservierten Präparaten nachweisen. Die dem Blutstrom anliegenden nackten Zellgruppen sind aussen ebenso scharf und glatt konturiert, wie die Zellen des übrigen Parenchyms. Ebensowenig merkt man etwas vom einem corpusculären Sekrete, das von diesen nackten Zellbezirken direkt in das Venenblut hinein abgestossen wird, wie es noch neulich von $\mathrm{Hultg}$ ren und Andersson geschildert wurde. Diese Autoren bilden in ihrer grossen Arbeit zahlreiche, bald innerhalb der Zellen, bald ausserhalb derselben gelegene Körnchen $a b$ und halten dieselben für Sekret. Wenn ich auch die Anwesenheit dieser Körnchen bestätigen kann, so scheint mir doch die Annahme, dass es sich um Sekretionserscheinungen handelt, schon aus dem Grund sehr unwahrscheinlich $z u$ sein, weil ja nur sehr kleine Zellbezirke 
direkt dem Blute anliegen und weitaus die grössten Strecken von dem Venenlumen durch die Gefässwand getrennt bleiben. Wir gelangten vielmehr auf Grund unserer Untersuchung zum Schluss, dass die Sekretionsfläche der Zellen an den gegenüberliegenden, der Lakune zugekehrten, kernhaltigen Hälfte der Zellen zu suchen ist. Die innere, sezernierende Fläche der Läppchen begrenzt also die bereits vorhin erwähnte unregelmässig gestaltete, mit dem Venensystem durch eigentümliche Gefässsprossen in Verbindung stehende Lakune.

Bei näherer Untersuchung derselben, namentlich auf tangential $\mathrm{zu}$ ihrer Oberfläche geführten Schnitten (Fig. 7), gewahrt man eine grosse Anzahl meist feiner, interzellulärer Gänge, welche sehr deutlich das nach Zimmermann für ähnliche Gebilde typische Bild aufweisen, nämlich schwarze (Eisenhämatoxylin)-Linien im Flächenbild der Wand, und Punkte im Querschnittsbild, als Abschluss der interzellulären Kittsubstanz gegen das Lumen hin (Schlussleisten). Diese interzellulären Gänge, welche sehr häufig anzutreffen sind, liegen vorwiegend zwischen den äusseren, antinukleăren, die konvexe Seite der Läppchen bildenden Abschnitten der Zellen. Es scheint allerdings, dass es sich um vergängliche, bald verschwindende, bald wieder entstehende und sich unter Umständen stark erweiternde Gebilde handelt, da sie sogar bei einem und demselben Tiere (Kaninchen) sehr stark, sowohl an Zahl wie an Grösse variieren. Da diese feinen Günge in die grossen, weiten, unten geschilderten Lakunen münden und in dieselben ziemlich allmählich übergehen, scheint hre Ausbildung mit dem Sekretionsstadium und der Sekretionsintensität innerhalb des Lüppchens zusammenzubängen. Sie werden wohl erst durch Dehiszenz der benachbarten Zellen behufs Abfuhr der Sekretionsprodukte der Zellen gebildet. Ebenso häufig wie zwischen zwei Zellen findet man solche Kanälchen auch in ansehnlicher Weite an Stellen, wo drei Zellen mit mehr oder weniger stumpfwinkligen Kanten zusammenstossen (Fig. $\mathfrak{T}, 10$ ). Diese Kanälchen, in deren Wand 2-3 mit Eisen-Hümatoxylin stets scharf und schwarz gefärbte Schlussleisten verlaufen, und welche dadurch von Rissen leicht unterscheidbar sind, sind also von sehr schwankender Grösse und verlaufen von der äusseren konvexen Oberfläche der Lüppchen, wo sie zuweilen unmittelbar unterhalb der Kapsel beginnen, gegen das Zentrum des Läppchens hin, wo sie sich in die Lakune ergiessen (Fig. 8, 10). 
Die, die Lakune begrenzenden Zellen sind ausnahmslos mit ihrer breiteren Seite in der Aussenseite des Zellgewölbes (Querschnittsbild der Markzylinder) gelegen, mit ihrem etwas verjüngten Ende der Lakune zugekehrt. Die Zellkerne, welche in der Regel exzentrisch liegen, befinden sich ebenfalls an der lakunären, sezernierenden Seite der Zellen.

Die eigentümlich unregelmässige, meistens etwas zugespitzte Form der lakunären Zellenseiten verlangt eine spezielle Betrachtung. Bald sehen sie wie angefressen aus, bald lösen sie sich in einzelne läppchenartige Fortsătze auf; dabei bleiben sie zưweilen mit einem langen ausgezogenen Fusse an der benachbarten Zelle oder an der Gefässwand angeheftet (Figg. 8, 11 u. 12). Im auffalligen Gegensatz zu dieser Unregelmässigkeit der freien Zellfläche steht der ausgezeichnete Erhaltungszustand des übrigen Zellenleibes und des Zellkernes. Dieses, sowie das Fehlen von Schrumpfungserscheinungen oder von Ablösung der Zellen von der Aussenkapsel der „Lappchen“, ferner die scharf konturierte Begrenzung der interzellulären Kanäle und der Wände der Lakunen lassen uns den Gedanken an ein etwaiges Kunstprodulkt als Folge der Fixation, an ein Abreissen der nukleären Zellseiten von den Gefässen u. dgl., überhaupt an eine künstliche Entstehung der Lakunen ausschliessen. Das unregelmässige Aussehen der nukleuren Zellflächen, ihr "Angefressensein" lassen vielmehr auf einen eigentümlichen, an dieser Stelle ablaufenden Sekretionsvorgang schliessen; es müssen die der Achse des Markbalkens zugewendeten Teile der Zellen bei der Sekretion einschmelzen und ihre Zerfallsprodukte müssen aus der Lakune durch den offen in sie mündenden Gefässspross weggeschafft werden. Das auf diese Weise gebildete Sekret lässt sich nun auch in vielen Fallen, aber durchaus nicht bei allen Tieren in Form feinster, im Lumen der Lakunen verteilter, mit Eisen-Hämatoxylin sich schwărzender Flöckchen nachweisen (Fig. 11). Diese Sekretflöckchen können natürlich möglicherweise erst durch Füllung bei der Fixierung entstanden sein; es lässt sich demnach nicht direkt entscheiden, ob auch das frische Sekret geformte Elemente enthalt; wir glauben immerhin, dass die von $\mathrm{Hultgren}$ und Andersson in den grösseren Venen im frischen Blut und in fixierten Präparaten nachgewiesenen Sekretpartikel mit den unsrigen identisch sein dürften. Die Ausstossung oder Sekretion 
derselben geschieht jedoch unserer Meinung nach nicht direkt aus den peripheren Teilen der Markzellen in die Venengefasse hinein, wie solches nach der Schilderung der skandinavischen Autoren stattzufinden scheint, sondern an der dazu speziell geeigneten, inneren lakunären, dem Kern benachbarten Sekretionsfläche und nach der Lakune hin. Es ist nun nicht daran zu denken, dass der Sekretionsvorgang mit einer vollkommenen Degeneration der Markzellen einhergeht. Abgesehen von ganz vereinzelt vorhandenen, degenerierten, vakuolisierten und in das Lumen der Lakunen abgestossenen Zellen (Fig. 8), scheinen die Zellen sowohl in ihrem ganzen Cytoplasma, wie auch namentlich im Kern stets wohlerhalten, ohne Spuren von Degeneration zu sein. Der Sekretionsmodus müsste demnach in einem nur partiellen Einschmelzen ihrer nukleären Seite und im Wiederaufbau des Eingeschmolzenen durch das intakt bleibende Cytoplasma und den Kern bestehen (merokrine Drüse Ranvier). Es verdient noch hervorgehoben zu werden, dass die oben erwähnten Sekretflöckchen nie innerhalb der engen interzellulären Kanälchen und Gänge gefunden werden. Ob man daraus den Schluss ziehen darf, dass das geformte Sekret nur von den angefressenen nukleären Flöckchen der Markzellen gebildet wird, und die seitlichen, einander anliegenden Flächen der Zellen in die dazwischen liegenden Kanachen nur flüssige Bestandteile sezernieren, bleibt immerbin dahingestellt. Die vorhin erwälnten, einzeln vorkommenden, degenerierten Zellen wurden bei einer Ratte in etwas grösserer Anzahl, teilweise noch im Verband mit den Läppchenzellen, meistens jedoch frei in der Lakune schwimmend vorgefunden. Über ibre Bedeutung konnten wir uns keine bestimmte Vorstellung bilden.

Die Beziehungen der Lakunen zum Blutgefässsystem wurden bereits im obigen geschildert. Das in der Lakune angesammelte Sekret wird wohl seinen Weg durch den in dasselbe mündenden Gefässsprossen nach der Vene hin einschlagen. Der Sekretionsdruck und die Anhäufung des Sekrets kann allerdings nicht sehr bedeutend sein, da eine vitale Tuschinfusion in die Aorta des lebenden Tieres schon genügte, um in einige, wenn auch nur vereinzelte Lakunen der Ratte Tuschpartikel einzuführen (Fig. 6). Auch findet man in vielen Lakunen des Kaninchens vereinzelte Blutkörperchen (Fig. 8), wobei allerdings das Vorwiegen der Archiv f. mikrosk. Anat. Bd. 63. 
Leukocyten ganz auffällig ist. Es dürfte sich daher vielleicht zum Teil um ein chemotaltisches, durch das Sekret hervorgerufenes, gegen den schwachen Sekretstrom gerichtetes Wandern derselben handeln.

Die Marksubstanz bietet uns, wie wir oben gesehen haben, ein exquisites Bild einer Drüse mit innerer Sekretion, ja strenggenommen das bis jetzt einzige in der Morphologie bekannt gewordene Beispiel derselben dar, da ja der Vorgang der inneren Sekretion bei vielen anderen Drüsen wohl eine physiologisch erschlossene, jedoch nicht eine morphologisch bewiesene Tatsache ist. Es ist nun von hohem Interesse, dass auch bei einer Drüse mit innerer Sekretion die für alle Drüsen geltende Eigentümlichkeit einer Polarität der sezernierenden Zellen, einer Trennung der dem zuleitenden Saftstrome zugekehrten und der sezernierenden Fläche durchgeführt ist, was ja für diese Art von Drüsen durchaus kein notwendiges Postulat ist: apriori musste auch mit der Möglichkeit gerechnet werden, dass die Sekretion durch Diffusion in dieselben Gefässe und an derselben Seite erfolgt, an welcher auch die Stoffaufnahme vor sich geht.

Wenn wir die Ergebnisse unserer Untersuchungen überblicken, so ist vor allem die Schlussfolgerung unabweisbar, dass eine tiefgreifende morphologische und physiologische Verschiedenheit $z$ wischen der Rinde und dem Mark besteht. Die feineren morphologischen Verhăltnisse, in Verbindung mit dem Ergebnis der vitalen Tuschinjektionen berechtigen uns zum Schlusse, dass wir es in der Rinde mit einem vorwiegend oder vielleicht ausschliesslich resorbierenden Organe, im Mark mit einer echten Drüse mit innerer Sekretion zu tun haben. Für beide Funktionen sind die morphologischen Vorbedingungen in einer ganz exquisiten Weise erfüllt.

Die Wahrscheinlichkeit dieser Schlussfolgerung ist ja schon durch die Tatsache des physiologischen Nachweises verschiedener Funktionen des Organes nahegelegt, es sind einmal entgiftende Funktionen anzunehmen, da die Erkrankungen oder das Feblen der Nebennieren zu schweren Autointoxikationen des Organismus führen. Diese Entgiftung könnte allerdings auch seitens der Nebennieren durch eine Ausscheidung von Stoffen erfolgen, welche die im Blute oder Organen vorbandenen Toxine neutralisieren; die experimentelle Entscheidung darüber, welche 
dieser Möglichkeiten in Betracht kommt, dürfte nicht allzu schwer fallen.

Es ist zweitens anzunehmen, dass von der Nebenniere bestimmte Stoffe dem Blut zugeführt werden, welche S te ig e r u n g des Blutdruckes bewirken, während sie vielleicht mit jener Entgiftung nichts zu tun haben. Die physiologischen Versuche ergeben nun in der Tat diese zweifache Tätigkeit der Nebenniere; es ergibt sich so von selbst die Frage, ob nicht diese doppelte Funktion auf verschiedene Bestandteile der Nebenniere verteilt ist. Für die blutdrucksteigernde Substanz ist das Mark als Lieferant verantwortlich $\mathrm{zu}$ machen. Abgesehen davon, dass in der Tat die Marksubstanz für eine Drüse angesprochen werden darf, welche wohl eine solche Substanz absondern könnte, erhellt dies ganz besonders aus den Versuchen ron $S$ wale Vincent, welcher grosse Drüsen von Rindenextrakt (bei Selachiern) den Tieren ohne jeden Erfolg injizierte, während kleine Dosen des Markextraktes Blutdrucksteigerung, grosse Dosen sogar tötlichen Ausgang zur Folge hatten.

Die Ergebnisse von $\mathrm{Hultg}$ ren und Andersson, welche auch getrennt Rinde- und Markextrakt injizierten und dabei nur qualitative Unterschiede in der physiologischen Wirkung (bei der Rinde viel schwächer) konstatieren konnten, fallen dem gegenüber weniger ins Gewicht, da sie, wie aus ihren Angaben hervorzugehen scheint, als Material Nebennieren von Warmblütern benutzten, bei welchen beide Schichten makroskopisch voneinander unmöglich scharf $z \mathbf{u}$ trennen sind, wo es daher gar nicht ausgeschlossen erscheint, dass mit der Rinde auch Markteile mitgenommen wurden. Die Experimente von Swale Vincent dagegen beziehen sich auf Selachier, bei welchen beide Abschnitte räumlich ganz getrennt sind. Dass für die eigentliche sekretorische Funktion der Nebenniere, die Ausscheidung eines blutdrucksteigernden Stoffes, die Marksubstanz auch in ihrer morphologischen Beschaffenheit in exquisiter Weise geeignet ist, ergibt sich aus der obigen Schilderung derselben.

In gleicher Weise stimmen mit der von $\mathrm{Swale}$ Vincent gefundenen Wirkungslosigkeit des reinen Rindenextraktes unsere Befunde über den Bau der Rinde überein, nach welchen die Funktion der Rinde garnicht in der Sekretion und Abgabe eines besonderen Stoffes in das Blut bestehen kann. Bleibt für die 
so mächtige Formation der Rinde eine drüsige Funktion ausgeschlossen, so werden dafür alle Einrichtungen derselben daraufhin, dass hier dem Kreislauf Substanzen entzogen werden. Dass es sich hierbei um eine Entgiftung des Organismus und um ein Unschädlichmachen der giftigen Substanzen handelt, ist allerdings nur eine der weiteren möglichen Annahmen. Soviel aber darf behauptet werden: Wenn überhaupt eine entgiftende Wirkung der Nebenniere besteht, so muss dieselbe der Rinde zugeschrieben werden.

Es erübrigt mir die angenehme Pflicht, meinem hochverehrten Lehrer, Herrn Professor Strasser, sowie Herrn Privatdozenten Dr. A. Gurwitsch für ihre gütige Unterstützung bei dieser Arbeit meinen ergebensten Dank auszusprechen.

Bern, Juli 1903.

\section{Erklärung der Tafeln XI und XII.}

Fixierung: Zeaker'sche Flüssigkeit. Färbung: EisenhämatoxylinRubin mit Ausnahme von 2, 3 und 4, welche mit Rubin allein gefärbt sind.

Fig. 1. Zwei aneinanderstossende Fasciculatazellen des Kaninchens. Beide zeigen eine Plasmaanhäufung zwischen dem Kern und der Stelle ihrer Berührung, an welcher sich eine gangförmige intercelluläre Dehiscenz mit stark färbbaren Wänden findet.

Fig. 2, 3. Partien aus den Fasciculata der Ratte. Vitale Tuscheinfusion (s. Textseite 7). C. = Intracellaläre Kanäle mit Tuscheinhalt, B. = wandlose Gefässstrecken, E. = vereinzelte Endothelzelle mit retrocellulärer Tuscheanhäufung.

Fig. 4. Schematische Umrisszeichnung des Markes eines Kaninchens (Querschnitt). Dunkelgrau die Markzellen, hellgrau die umgebende Rindenpartie, weiss die Venen; schwarze Linien: die Grenzen der Markzylinder resp. Venenwände und Bindegewebssepta, rot: die intierbalb der Markzylinder gelegenen Gefässe und Bindegewebszüge; ${ }^{*}=$ Kommunikationsstellen der letzteren mit den Venen; die weissen Aussparungen innerhalb der Markbalken deuten die Lakunen an. † Bindegewebssepta sind schmale Ausläufer der venösen Sinuse zwischen den Markzylindern. Die Konturen der Venen und der Markzylinder, die Stellen *, die Biadegewebssepta und die schmalen Ausläufer der venösen Sinus zwischen den Markzylindern sind genau nach dem Präparat eingezeichnet, das übrige schematisiert. 
Über die Beziehungen zwischen dem Blutgefässsystem etc.

Fig. 5. Zwei Zellen aus dem Marke des Igels, mit Sphäre und Diplosoma.

Fig. 6. Markläppchen einer Ratte (vitale Tuscheinjektion), die interlobulären, spaltförmigen Gefässe, dicht mit Tusche gefüllt. Tuschepartikel im wandlosen lakunären Gefäss und zwischen den Zellen.

Fig. 7. Tangentialschnitt durch ein "Läppchen". Zahlreiche zwischen zwei und drei Zellen gelegene intercelluläre Kanälchen.

Fig. 8, 9. Zwei aufeinander folgende Querschnitte durch einen Marbbalken ${ }_{\text {"Läppchenbild }}$. G. $=$ in die Lakune offen mündender Gefässspross. In Fig. 8a der Schnitt durch den Gefässspross der Fig. 8 bei tieferer Einstellung.

Fig. 10. Schnitte durch zwei benachbarte Markzylinder mit unregelmässigen Lakunen. G. = Mündung eines Gefässsprosses in die Lakune, $\mathrm{L}_{\mathrm{C}}=$ wandlose Strecke einer kleineren interlobulären Vene, A. = Querschnitt durch einen Ausläufer des Lakune. Intercelluläre Kanälchen.

Fig. 11, 12. Tangentialschnitt durch Markzylinder. Unregelmässige dusläufer der Lakunen; in 11 feine Flocken (Sekret?) im Innern der lakunären Räume.

\section{Literatur.}

A lexander, C.: Untersuchungen über die Nebennieren und ibre Beziehungen zum Nervensystem, in Zieglers Beiträge 1893, Bd. 11.

B ro.wicz: Jleine Ansichten über den Bau der Leberzelle, in Virchow's Archiv 1903.

Ci a c c i o, C.: Im Anatomischen Anzeiger 1903.

Carlier, E.: Note of the strukture of the suprarenal body, im Anat. Anz. 1893, Vol. VIII, No. 12, 13.

Félicine: Anat. Anz., Bd. 22, 1902.

Flint, Jos. Marsh.: The Blood-ressels, angio genesis, organogenesis, reticulum and histology of the adrenal. Vol. IX of the Johns Hopkims Hospital Reports.

Gottscha a, M.: Über die Nebennieren der Säugetiere, speziell über die des Menschen, im Sitzungsbericht der phys.-med. Gesellschaft zu Würzburg 1882, Nr.4.

Derselbe: Struktur und embryonale Entwicklung der Nebenniere bei Säugetieren, im Archiv für Anatomie und Physiologie, Anat. Abt. 1893.

Gu i y s se, A.: Etudes sur la capsule surrénale de la cobaye, in Journ. de l'anatomie et de la physiol. 1901.

Gurwitsch, A.: Zur Morphologie und Physiologie der Nierentätigkeit, in Ptliugers Archiv 1902.

H ol m gren: Ergebnisse v. Merkel n. Bonnet. 1902.

Hultgren, E. O. und Andersson, 0.: Studien über die Physiologie und Anatomie der Nebennieren, in Skandinavisches Archiv für Physiologie 1899 . 
312 Lydia Félicine: Über die Beziehungen zu dem Blutgefässsystem etc.

จ. Kupffer: Über die Sternzellen der Leber, im Archiv f. mikr. Anat. Bd. 48 .

M a nasse, P.: Über die Beziehungen der Nebennieren zu den Venen und dem venösen Kreislanf, in Virchows Archiv 1894, Bd. 135.

Mulon, C. R.: Soc. Biol. Paris 1902.

Overton: Jahrbuch f. wissensch. Botanik 34, 1900.

Derselbe: Vierteljahrsschrift d. Naturforschenden Gesellschaft Zürich Bd. $40,44$.

Roud, A.: Sur le développement de la glande surrénale de la souris. Lausanne 1902.

$\mathrm{Swale}$, Vincent: The effects of subcutaneous injections of extracts of suprarenal capsulus, in Journ. of phys. 1897, Vol. 21.

Derselbe: On the general eftects of extracts of the suprarenal capsules in Journ. of phys. 1897, Vol. 22. 